\title{
A weight-aware recommendation algorithm for mobile multimedia systems
}

\author{
Pedro M.P. Rosa ${ }^{\mathrm{a}}$, Joel J.P.C. Rodrigues ${ }^{\mathrm{a}, *}$ and Filippo Basso ${ }^{\mathrm{b}}$ \\ ${ }^{a}$ Instituto de Telecomunicações, University of Beira Interior, Covilhã, Portugal \\ ${ }^{\mathrm{b}}$ Zirak s.r.l., Italy
}

\begin{abstract}
In the last years, information flood is becoming a common reality, and the general user, hit by thousands of possible interesting information, has great difficulties identifying the best ones, that can guide him in his/her daily choices, like concerts, restaurants, sport gatherings, or culture events. The current growth of mobile smartphones and tablets with embedded GPS receiver, Internet access, camera, and accelerometer offer new opportunities to mobile ubiquitous multimedia applications that helps gathering the best information out of an always growing list of possibly good ones. This paper presents a mobile recommendation system for events, based on few weighted context-awareness data-fusion algorithms to combine several multimedia sources. A demonstrative deployment were utilized relevance like location data, user habits and user sharing statistics, and data-fusion algorithms like the classical CombSUM and CombMNZ, simple, and weighted. Still, the developed methodology is generic, and can be extended to other relevance, both direct (background noise volume) and indirect (local temperature extrapolated by GPS coordinates in a Web service) and other data-fusion techniques. To experiment, demonstrate, and evaluate the performance of different algorithms, the proposed system was created and deployed into a working mobile application providing real time awareness-based information of local events and news.
\end{abstract}

Keywords: Mobile computing, ubiquitous computing, location-aware, content-aware, iphone applications, multimedia applications, mobility

\section{Introduction}

Utilization of mobile devices in everyday life has expanded rapidly over the past few years; consumers are changing their habits by using resources offered by the Internet, that is now always available in a click. Content-adaptation and context-awareness are more and more necessary, to be able to deal with the current information flood provided to the user [14].

The use of smartphones all over the world is growing rapidly, with a large adoption rate especially among teenagers and adolescents [35]. Mobile devices can nowadays natively support several kinds of multimedia, and mobile services are supporting the users with an exponential growth of specialized applications/services for almost every need: as a general thermometer can be used the number of deployed applications in the main mobile Markets/Stores present in Internet. The expression "Information at your fingertips anytime, anywhere" has been driving the mobile computing development in the past two decades. However, mobile devices do not have the same features in what concerns to conventional information processing, such as PC's and laptops [21], especially in terms of computing power,

\footnotetext{
${ }^{*}$ Corresponding author: Joel J.P.C. Rodrigues, Instituto de Telecomunicações, University of Beira Interior, Rua Marquês D’Ávila e Bolama, 6201-001 Covilhã, Portugal. E-mail: joeljr@ieee.org.
} 
human-machine interaction (HMI) resources, general limitations of network, battery cycle, and other specific topics, becoming important limitations in mobile computing [23]. The rise of smartphones like the Blackberry, Android, and iPhone allow not only voice communication but also communications via SMS (Short Message Service)/MMS (Multimedia Messaging Service)/E-Mail/Social Networks. They also have the capacity to process intensive activities such as multimedia playback, document editing, and audio/video streaming via dedicated coprocessors. In spite of the inherent limitations, some authors are supporting the idea that conventional laptops will be replaced by smartphones and tablets soon [33].

Mobile devices provide a wide range of opportunities to a global society through online social networks, blogs and web pages, among others. New approaches to access information in mobility are being improved; over the last years the research community has studied and developed new technologies, services and applications to enable ubiquitous environments based on mobile technology [38]. The new generation of mobile devices has improved the efficiency of representation of the information, enhancing user experience [5]. Ubiquitous computing is becoming a reality with mobile computing, due to a rapid advance in wireless technologies and Internet [35].

The small screen size of the mobile phone does not help to read contents. It is difficult to present news efficiently or to display only the context of mobile news and events, capturing users' interest. Despite all the effort made to contribute to improved navigation and usability in mobile news, results should be improved constantly, not to decrease in popularity among users and result in a poor experience while navigating the mobile news [21].

Applications based on geo-location can benefit of a more stable Internet connection, enabling the user to access a wide range of server-side location-based contents, such as transport timetables, open restaurants or event calendars. Location-awareness plays an important role in the jungle of the contextawareness parameters, and everyday there are new apps using the global positioning system (GPS), its services and new features [38].

Joining ubiquitous mobile devices and web services brings into the user's hands the best of both worlds: resources and processing algorithms are server side while content-adaptation and direct contextawareness parameters are on client side [15]. The application focuses on what, where and how the users want to see and interact with the content provided. In general native applications produce better results than multi-platform solutions, contributing to a satisfactory exploration of cultural events fully utilizing the resources of the device [4].

In this scenario, recommendation systems are important to help a user to make choices, identifying the best news in an ocean of potentially good ones. For every specific application should be identified several parameters, or relevance, that can influence the filtering/ordering choices, in order to provide to the users the more important information. In some content-adaptation methods where some hundreds of hits are possible to be meaningfully shown, like a map with clustered points, it is important to maximize relevance to have at least 100-200 results; in other methods of data-presentation, like the list of best sold apps in AppStore, it is very important to have very high precision in the first 10 hits. The main issue is to identify the best parameters and the best measures to be optimized in order to them in the specific recommendation system. It is assumed that does not exist the best system, but different tunings depending on results the system is focusing on.

To provide an environment for experimenting, demonstrating, and comparing results coming from different algorithms, a dedicated application to provide news and events to a generic user was created. The results are ordered taking into account parameters coming from habits and instantaneous position. The habits chosen for this solution are the frequency of visits of a pre-defined taxonomy structure and the active sharing on social networks elements of the same taxonomy. 
Nonetheless within the same technological framework several other parameterizations, both direct (background noise volume) and indirect (local temperature extrapolated by GPS coordinates) can be considered. Relevance coming from these three context-awareness parameters are then combined, or fused, with some classical data-fusion techniques, to evaluate different ways of producing an ordered list of results. The following data-fusion methods are used and experimented: $i$ ) CombSUM, weighted CombSUM; and ii) CombMNZ, weighted CombMNZ. One of the important steps leading to a successful data-fusion is the re-normalization process that was studied case by case, for each one of the three relevance cases.

The main contributions of the paper include the study and the construction of a complete generic recommendation system, with both client mobile application and Internet service, and the verification of several re-normalization and data-fusion techniques applied to the specific case of news and events recommendation system [32].

The remainder of the paper is organized as follows. Section 2 elaborates on the related work about mobile recommendation systems. Section 3 gives a mathematical overview of the used re-normalizations and data-fusion techniques. Section 4 focuses on the application demonstration and validation. Section 5 gives a performance evaluation and results of the proposed application. Finally, Section 6 concludes the paper and pinpoints directions for future work.

\section{Related work}

The possibility of materializing the vision of ubiquitous computing that was drawn at the beginning of the 90's is approaching [29,36], combining new features of mobile devices with the growth of shortrange ad-hoc networks. In the early 90's, Marc Weiser [26,27] introduced his vision of ubiquitous computing. He presented a concept of a man-technology interaction with a complete abstraction of the user. However, Weiser's vision faced several problems, mainly lack of technological support [27]. A good example of ubiquitous technology is the Internet; users are not interested in the underlying technology behind the Internet, but only in the information and all the services provided by it [35]. Users are connected everyday to several social networks, without knowledge of protocols and network architectures used on a mobile device [28].

Mobile or portable devices such as mobile phones, personal digital assistant (PDA), and tablets are smaller and lighter, can be transported anywhere, and can easily fit in the suit pocket or briefcase. These portable devices have a good number of features, such as SMS (short message service), eMail, packet switching for access to the Internet, gaming, Bluetooth and Wi-Fi connectivity, infrared, photo camera and video recording, music player, radio and GPS antennas, memo recording, and, more importantly, make and receive phone calls. Mobile devices offer the opportunity to create a better and fast growing globally connected society, with social networks, blogs, and Web pages. These new approaches improved user-access to information through mobile communication $[24,47]$.

The research community has been studying and developing new technologies, new services and new applications over the years to enable ubiquitous environments based on the mobile technology [38]. Mobile devices improved communication efficiency, enhancing user-experience [19]. The exponential growth of people using mobile devices leads to a constant improvement of smart communications.

The ubiquitous collaboration between mobile devices and Web services brings the best of the two worlds: the server side resources and the client-side context and location [36]. The application is centered in the user preferences (where, when, and how), providing context and content-awareness. The 
information and context of the events on a native application offer a better visualization to users, contributing to a satisfying physical exploration of cultural and information events [9].

Ubiquitous computing is one way to improve the computers usage [39]. The main goal of ubiquitous computing turns human-computer interaction invisible, fully integrating the computer with the actions, and behavior of its users [40]. Computer systems that surround us are proactive and are linked together, or trying to establish links between themselves constantly. Ubiquitous computing requires Internet connectivity and this feature is often used and characterized for ubiquity [43].

Geo-location applications are becoming very useful due to anytime and anywhere full connectivity. Smart phones with GPS capability are becoming more widespread. This ability of smartphones can be used as a personal navigator and a communicator device. There are various mobile navigation techniques to determine the location used on mobile multimedia applications [30]. Due to their portability and vast range of applications, mobile devices are being appointed as the future Internet navigation devices. Native applications offer a good support for multimedia contents. This access is made using Internet services in native applications [30]. By adopting these techniques and concepts it was possible to develop an intelligent mobile multimedia application.

The context-sensitive computing (Context-Aware Computing) has emerged as the field of ubiquitous computing, studying the relationship between changes in the environment and information systems [2], and thus raising new technical challenges for implementation. In the computing context-sensitive computing (Context-Aware Computing) devices try to understand and to automatically capture the context where they are inserted. Offering a better interaction between the environment and the user. This interaction can happen in terms of hardware, software or communication [1].

In recent years, many platforms have been developed for pervasive and context-aware systems in order to support rich contextual features. Many of these systems are open and available [7]. An important aspect of mobile devices is their portability and mobility. With the advent of wireless communication in mobile phones, smartphones, and tablets, ubiquitous computing has evolved as well. They take advantage of their portability to have wireless connectivity almost everywhere [24]. In mobile computing there are some technological hurdles to overcome, such as the variation on the quality of a wireless network, local access limitation, and energy constraints. These problems affect the user and also the computing experience directly [4,12].

Mobile devices with GPS (Global Positioning System) capabilities have been around for some time. Location-awareness in mobile and other devices is changing the users life [38]. Devices with such capabilities and location-aware applications will lead the mobile market [3], such as pinpointing the location on a Google Map, tracking friends, a geo-located event, giving the idea on what is going on in the user area. They can tell us what is the nearest place to eat, giving a list of all shopping or stores in the user area, tell us where to go for a party or cultural events, and other businesses [13,17,25].

Data fusion algorithms deployed (CompSUM, CompMNZ and their relative weighted version) are already found in the works of Salton and Fox [16] and improved by Lee's article three years later [25] where it is shown that in specific situations of information retrieval data-fusion it is very important the renormalization phase, not to incur in wrong evaluations of the best methodology. Several articles are proposing new methods, more complex and adapted from other branches of knowledge like neural nets, algorithms of democratic voting from the Social Choice Theory, logistic regression formulas. Other fusion techniques applied to other scientific areas shows new simple ideas as outperforming all other state-of-the-art techniques, because of new boundary conditions, and with no answer to how and which conditions act on the effectiveness of a specific technique, except generic statements [22]. Analyzing several results, it become clear that the classical CompSUM and CompMNZ are enough simple and 
effective, compared to other specialized formulas, and the work was focused on them. Renormalization techniques investigated were based on simple linear transformations and an exponential transformation in the case of location-based parameters. Several parameters are found in the literature, and mobile information retrieval is maybe the more creative and rich in relevance parameterizations; as an example, in "Music Recommendation Using Content and Context Information Mining" [39] uses context conditions such as location, time, air temperature, noise, light, humidity, and motion to make music recommendations that are sensitive to the user's mood [14]; another interesting approach can be found in [8], where a Movement Pattern Mining is determined starting from a User Movement Database (UMD), determining on a statistical base (that for data-fusion can be individual and/or collective) the patterns and distribution of future positions of the user.

Inside the Apple App Store users can find location-aware applications, such as Loopt-Friendfinder [18], application with virtual earth display that, allows user to share their location with the community. NearPics [41] is a location-aware photo browser, and Weatherbug [45] is a location aware weather service with predefined cities. Appetite [3] is a restaurant picker based on user location; AroundMe [42] is an application that gets your location and allows you to choose the nearest bank, bar, gas station, hospital, hotel, movie theatre, restaurant, supermarket and taxi. Every day a new item is added to this list.

An application for iPhone, called N4MD - News for Mobile Devices, is presented in [19]. This is an application to visualize the weekly news produced at the Urbi et Orbi newspaper from the University of Beira Interior, Portugal. The application N4MD runs natively on iPhone devices and now, it is similar to hundreds of newspapers and magazines mobile applications. The system proposed in this paper is composed by CityEvents app and an online recommendation service that is unique on its way of gathering an arbitrary set of relevance parameters, merging them into a personalized and meaningful global relevance able to filter and order events and news to fit the needs of a generic recommendation system. Several algorithms were evaluated and compared, and based on revised scientific knowledge, it is proposed a new approach that is able to investigate with little efforts other more recent algorithms and parameters.

\section{Weighted scoring algorithm for mobile information applications}

Considering a system with $N$ meaningful parameters that expresses some kinds of context-awareness relative to a specific user, and where, out of a huge number of atomic informations (news, events, or more in general documents), some information should be provided to the user sorted and/or filtered in order to maximize precision. A general system require a parameters re-normalization phase, where the parameters are meaningfully mapped to the $[0,1]$ interval, and a data-fusion phase, where the $N$ parameters are joint into a single one, from which ordering and/or filtering can be provided, dependent to the specific content-adaptation provided by the mobile device. Weights are provided to allow user requests personalization, and real-time perception of the differences that can occur if, for example, he/she considers location to be more important than habits or vice-versa.

In this work, the following three parameters were analyzed:

1. Location-based scoring, giving a score relative to the Euclidean distance between the user and the position of the news/event; other types of distance can include the path distance following roads or cycle-paths, or estimated time for a specific vehicle to move from the user to the news/event position. 
Table 1

Location-based scoring parameters

\begin{tabular}{lcl}
\hline Transport mode & $\mathrm{D}$ & $\alpha$ \\
\hline Walking & 2.000 & $2 / 2.000$ \\
By bicycle & 12.000 & $2 / 12.000$ \\
By car & 40.000 & $2 / 40.000$ \\
\hline
\end{tabular}

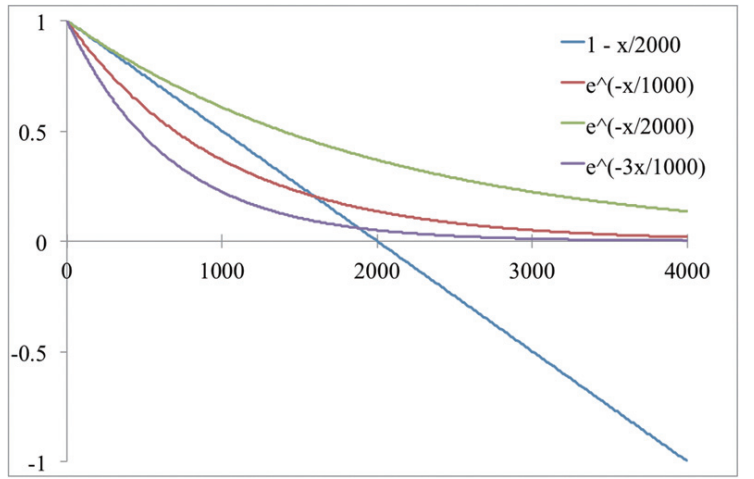

Fig. 1. Effect of different parameters in Location-based scoring.

2. Habit-based scoring, the frequency of visits of a pre-defined taxonomy structure; in this case a flat categorization was a sufficient approximation, but a more complex structure provided with an internal metric will benefit of the same amplified benefits.

3. Habit-based scoring, the active sharing on social networks of elements of the same taxonomy structure; assuming a correlation between active sharing and interest, several measures can be built to provide meaningful relevance; in this case, to simplify, it was used the frequency of article sharing, grouped by the same flat categorization of parameter 2 .

\subsection{Location awareness scoring}

Given the Euclidean distance $(d)$, in meters, between a device and an event, let $s_{d}$ be the distance scoring; a simple scoring, useful for this approach, can be defined as follows:

$$
\begin{aligned}
& S_{d 1} \equiv \max \left(\left(1-\frac{d}{D}\right), 0\right) \\
& S_{d 2} \equiv e^{-\alpha d}
\end{aligned}
$$

To define the constants $D$ and $\alpha$ it can be noticed that they identify different exclusive scoring functions with a specific physical meaning for a user that commonly uses a bicycle (or a car, or just walks around the city), it is important to distinguish between events that are $100 \mathrm{~m}$ or $300 \mathrm{~m}$ away from the current position, while it is not so important if they are at $30 \mathrm{~km}$ or $100 \mathrm{~km}$ away; the same can be false for a user that commonly uses a car. Table 1 presents these constant values defined empirically, based on user feedback from a dozen of users.

Taking into account the scoring system used by the user that is commonly walking, Fig. 1 highlights how the factor $D$ represents the value where $s_{d 1}$ gives zero scoring, thus considering identical all the events that are more than $2 \mathrm{~km}$ away from the user.

From Table 1 it can be seen that good empirical values for $\alpha$ can be obtained with the equation $\alpha=2 / D$, to keep scoring and its derivative not too different from the linear case in $[0, D]$ and being not too high when $S_{d 1}=0$. A factor 3 (green line, Fig. 1) should give too much importance to nearby events (being the derivative much smaller for small distance), while a factor 1 would behave like the linear function only for nearby events, but will be too high for events with distance $D$ or bigger.

In the first deployments of the algorithms it was experimented accurately the first scoring system on Eq. (1), while after some intensive experiments was noticeable the different behavior with situations that 
does not use the awareness scoring system, but only the location awareness scoring. Thus, it was chosen a more valid approach following the second scoring system given by the Eq. (2). It is very simple, easy to manage, monotonically decreasing, and with not appreciable changes in computational time.

Other meaningful parameters can be used, such as time estimate instead of distance, but in the current approach only the above-described exclusive distance functions were selected.

\subsection{Habit awareness scoring}

While the described location awareness scoring is mainly one-dimensional (having different exclusive scoring equations for different transportation habits), the deployed context awareness scoring is multidimensional, giving different meaningful scorings to measure how a person can be interested in some context more than others. In this case two relevant parameters were selected. One is based on the frequency of seeing the details or sharing social networks news or events belonging to a specific taxonomy. Another parameter is based on a flat categorization, but it can be easily extended to a generic taxonomy provided with a good metric [31].

In general, assuming $N$ different non-negative context-based relevance, $S_{c}=\left\{S_{c 1}, S_{c 2}, \ldots, S_{c N}\right\}$, it is important to keep in consideration the possible correlation between them, and remap the values of every relevance in the interval $[0,1]$, similarly to the approach followed on the location scoring. The correlation between the relevance can be dealt with appropriate weights in an upcoming phase, when a total scoring should be defined through a data-fusion algorithm.

To re-normalize the values, the function $s_{c j}$ as function of a discrete set of information was considered, $J=\left\{j_{1}, j_{2}, \ldots, j_{M}\right\}$. Thus, with limited values, remapping a simple linear mapping is shown in Eq. (3).

$$
\left\{\begin{array}{l}
s_{c j}^{\prime}(0)=0 \\
s_{c j}^{\prime}\left(\max \left\{s_{c j}(I), c_{c j}\right\}\right)=1
\end{array}\right.
$$

The $c_{c j}$ values are empirically defined as minimum values that depends on the context based relevance, and represent the minimum scorings that can be considered valuable (for example, if $s_{c 1}(k)$ represents the number of times that an user shared information of the category $k$ via a social network, $c_{c 1}$ can be set to a value around 15-20 units); in the extensive performed experiments, this lower limit helped to obtain a more precise global relevance in those cases where the specific scoring method was under-utilized.

\subsection{Comparison of habit-based and location scoring}

Comparing habit-based and location-based relevance, it is immediately noticeable a difference: the maximum value 1 is often present in the image of every non trivial element of $s_{c}$, if the set $I$ is non-empty and $c_{c j}$ properly set, while it is almost always absent in the image of $s_{d}$. Even if a simple linear remapping can be used to correct this different behavior, it was observed empirically that this difference could be positive for the global relevance. The underlying reason is that if there are no nearby informations, in general, it is more important the habit-based scoring.

In the current deployment several habit-based scoring parameters and three exclusive scoring parameters for location-based scoring (depending on main transportation method: walking, bike or car) were used. Other useful parameters that could be considered will be suggested for future work, such as the following:

- Frequency of "page-views" and/or "clicks";

- Frequency of "sharing" on a social network, grouped by social network; 
- Frequency of "send-to" specific users (the user forwards the information not because is interested, but because the target user is interested in the topic);

- Frequency of "like" or other feedback systems (percentage of the details shown, if the content is presented in scrolling or tree views);

- Specific user search terms.

The parameters could be grouped as follows:

- Inside a fixed taxonomy with a metric;

- Through document similarity, using the text in title and document body, after stop-words removal and stemming;

- By information-provider, or other natural groups.

The relevant parameters should become more complex when a bigger set of information flooding is needed; for example, time or day dependent parameters can give better results (the preferences of a user should change between working hours and non-working hours, between a week-day or weekends), media-content analysis can give new ranges of context awareness scoring, and semantic Web services can be used to provide online deep analysis, giving scope to the user to define his/her own areas of interest, that will reflect similar structure of his/her "to do lists", the eMail inbox, the documents organization, as well as the informations scoring.

\subsection{Combining context and location scorings}

In order to produce a single global relevance, out of a set of $N$ parameters is usually mentioned as "data-fusion". Several algorithms on data fusion topic were proposed, most of them more complex outperforming the more simple ones just in very specific conditions. It is not observed any advantage for the current proposal the use of more complex and processor intensive algorithms. Then, this work focuses in four main intuitive algorithms described in the classical work of Fox and Shaw [11]: CombSUM, Weighted CombSUM, CombMNZ, and Weighted CombMNZ.

These methods use relevance and not just their induced ordering (rankings), and they are defined as follows. For a document $d$, in our case an event or a news, and a set $S=\left\{s_{1}, \ldots, s_{N}\right\}$ of different relevance parameters, let $n_{d}$ the number of systems that returned positive relevance for a document $d$, and let $W=\left\{w_{1}, \ldots, w_{N}\right\}$ a set of weights that expresses not only user preferences but also the independence of the parameters, it is commonly defined as presented in Eqs (4)-(7).

$$
\begin{aligned}
\operatorname{CombSUM}_{s}(d) & =\sum_{i} s_{i}(d) \\
\operatorname{CombMNZ}_{s}(d) & =n_{d} \sum_{i} s_{i}(d) \\
{ }_{\operatorname{CCombSUM}}(d) & =\sum_{i} w_{i} s_{i}(d) \\
{ }_{\mathrm{CCombMNZ}}(d) & =n_{d} \sum_{i} w_{i} s_{i}(d)
\end{aligned}
$$

From Lee's works $[14,25]$ it was shown that data fusion techniques could be applied to combine different relevance, but only when the relevance is appropriately re-normalized (and the process of renormalization can influence the results of the data fusion itself). In his work, CombMNZ presented by Eq. (5) performs better than CombSUM - Eq. (4), but, in this proposal, it was noticed that both are very 


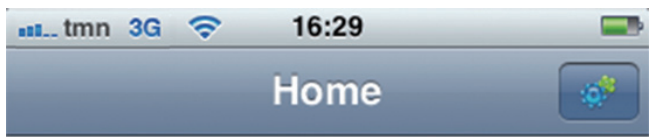

Information about culture events and local news

Featured Event

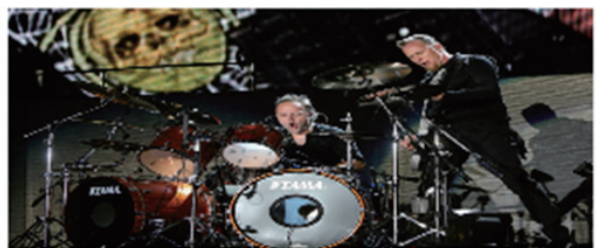

METALLICA CONFIRMED AT ROCK IN RIO LISBON-2012

After a spectacular concert at the Rock in Rio 2011 in Rio de Janeiro, Metallica return to the Portuguese edition of the biggest event of music and ontertainment world whore they

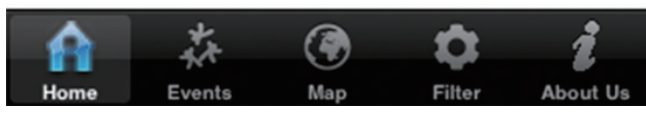

Fig. 2. Home Window.

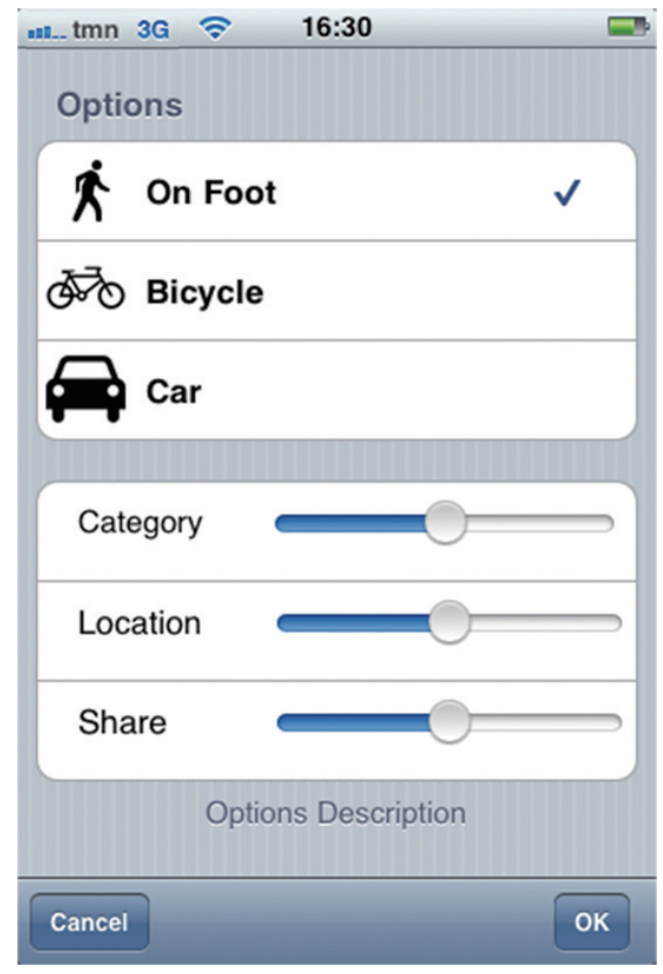

Fig. 3. User preferences screen.

similar and one does not outperform the other in all the situations, being easy to produce common cases where CombSUM, Eq. (4), or CombMNZ, Eq. (5), are better one another. CombSUM, Eq. (5), it is an algebraic mean, except a re-normalization factor $1 / N$, and thus represent a stable fusion technique, quite intuitive in the results, and well behaving in our experiments.

CombMNZ, Eq. (4), it is like a class-algebraic mean where the number of non-zero parameters defines classes and their relative weights. For example, in this proposal all the documents with 1, 2, or 3 non-zero parameters were grouped.

Weighted fuses, presented in Eqs (6) and (7), can have a big impact in mobile recommendation systems because they can give personalized results, depending on parameters that user can define in real-time, and perceive the feedback of this change (while statistical measures changes have usually a more stable behavior). The user can decide that these choices should be defined more by location (for instance, in several days/hours where he/she sees that there is more traffic), while it can be defined more by interests and habits in other days/hours. Asking feedback to users was noticed that using weights in the range $[0.33,1]$ gives best empirical results for users that provide significant relevance in all the 3 included parameters, probably because it will keep some balance between the extremes of founded values in a neighborhood of zero and others in a neighborhood of 1 .

\section{CityEvents demonstration and validation}

CityEvents is a simple mobile application for viewing events or news. The CityEvents system includes a user-friendly layout using the user-interaction capabilities of the iPhone. The user's interface is shown 


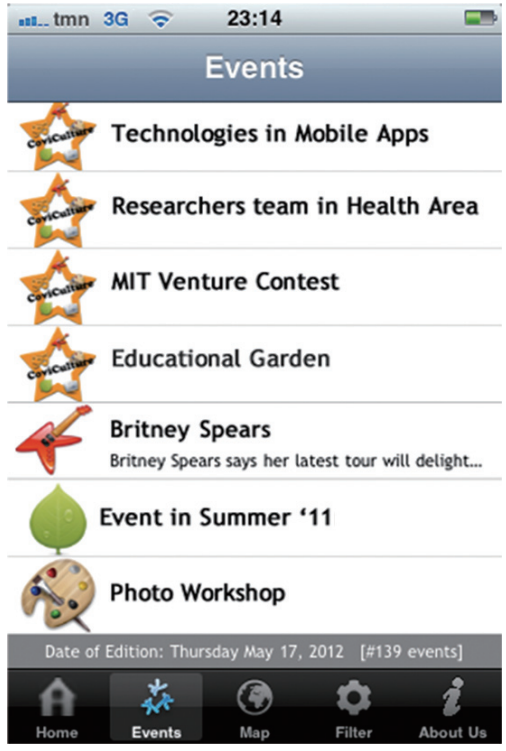

Fig. 4. List of all the available events.

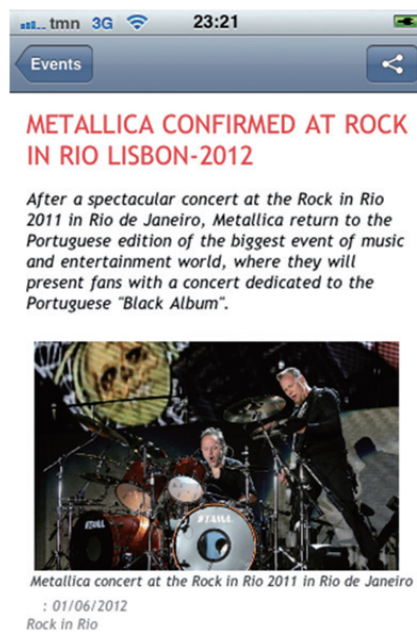

(a)

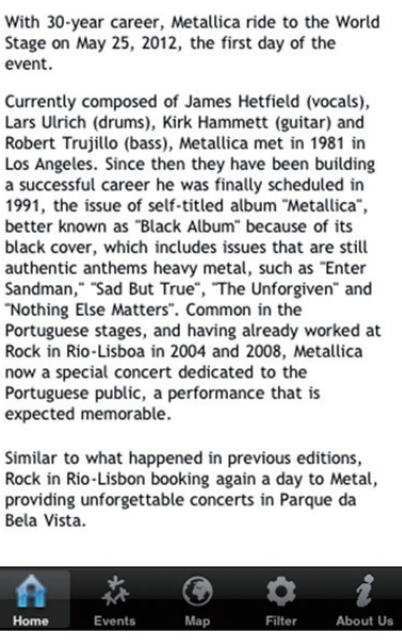

(b)

Fig. 5. Example of an event detail.

along the CityEvents system demonstration presented in this section. Figure 2 shows the Home window of the application. This window displays several informations (e.g. title, image, and a brief summary) about the week event, which is the most important category for the user. By clicking on the event image, the application leads the user to the detail page of the event. This feature allows users to know which event is considered the main week event, without the need to search it.

On the right top, a button for the User Preferences is available. Figure 3 displays all the preferences for the user, including the user's means of transportation and some criteria for being used. Those changes will affect the scoring of the events. Depending on user choices and preferences the results are shown on a map and a list of events. The map shows the location of the events. The most relevant events are displayed with colored icons while the less important have a black icon. There are icons for each category of events. This list of events is sorted from the most important to the less important ones.

At the tab bar Events, a list of events is shown as may be seen in Fig. 4. Displaying a list of events sorted by the user's preferences. At the bottom of this tab, the user can see the number of events available on the list. In the specific case shown in Fig. 4, the user may choose from a range of 139 events.

When an event of the list is selected, the system will get the corresponding information about it showing the details to the user (as shown in Fig. 5). As may be seen in Fig. 5(a), the details include the event title, a brief summary, an image with the corresponding caption, the event date, the publication date, and the author of the post. The description of the event can be found at the end of the view (Fig. 5(b). When the description field is bigger than the available window area, the user is allowed to scroll the text in order to read it.

At the Filter tab shown in Fig. 6(a), the user may choose a specific category of events. Figure 6(a) also presents a list of the available categories to the user. Figure 6(b) shows the result when the user chooses a category. The application will fetch only the user's choice and the information of these events. With this feature, CityEvents implements a cognitively distinct group of users.

At the Event Detail window the user has the possibility to share an event with friends, by tapping the share button. Three options are available (Fig. 7): sharing an event in the user Facebook page, sending it by email, or sending a text message. 
Table 2

Questions in CityEvents survey

\begin{tabular}{cl}
\hline Question & Description \\
\hline Q1 & Is the application design attractive? \\
Q2 & Is the application easy to use? \\
Q3 & Is the application environment user friendly and intuitive? \\
Q4 & Are the navigation options clear and consistent? \\
Q5 & Are fonts easy to read on the screen? \\
Q6 & Is the feedback and response time of the application fast enough? \\
Q7 & Is the application store with users' preference useful? \\
Q8 & Is the events/news sorted in function of users' preferences useful? \\
Q9 & Is the map with all the points of interest and events based on users' preferences useful and attractive? \\
Q10 & Is the events/news scoring based on users' preferences and location useful? \\
\hline
\end{tabular}

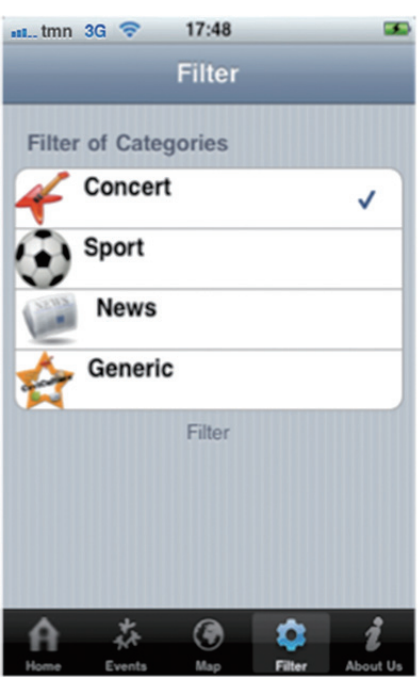

(a)

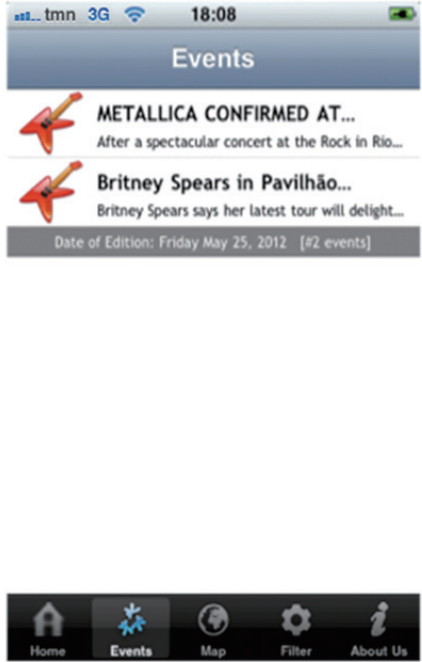

(b)

Fig. 6. List of categories and corresponding events.

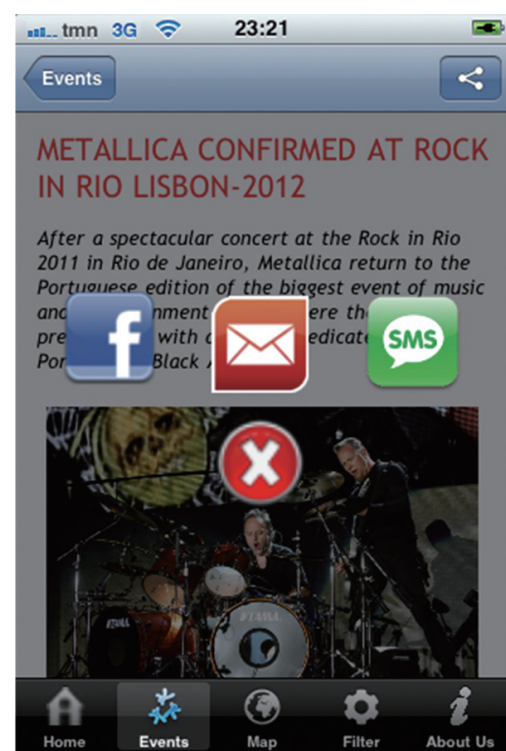

Fig. 7. Options to share events.

Figure 8 shows an example of a shared event. At the left inside, it is shown the event share on Facebook while at the right inside, the event is shared by email. On the lower portion of Fig. 8, the use of SMS is illustrated. It can be noticed that with these sharing features an event will receive a bigger score on the user's profile.

Figure 9 shows the Map tab presenting a map with the localization of an event. It is also shown all the close available events, using pinpoints to identify the event location. The nearby event is represented by the corresponding icon category. The user location is also shown on the map. When a category is selected, the user can read the title and a brief description of the event. Figure 9(a) shows an alert of the most relevant event to the user, catching his/her attention for an event of her preferred category. Figure 9(b) presents a map with user's preferred events, based on the most important preferences and categories to him/her.

The CityEvents system was evaluated in several mobile devices (iPhone and iPad) and performed very well, as expected, and it is ready for use. 


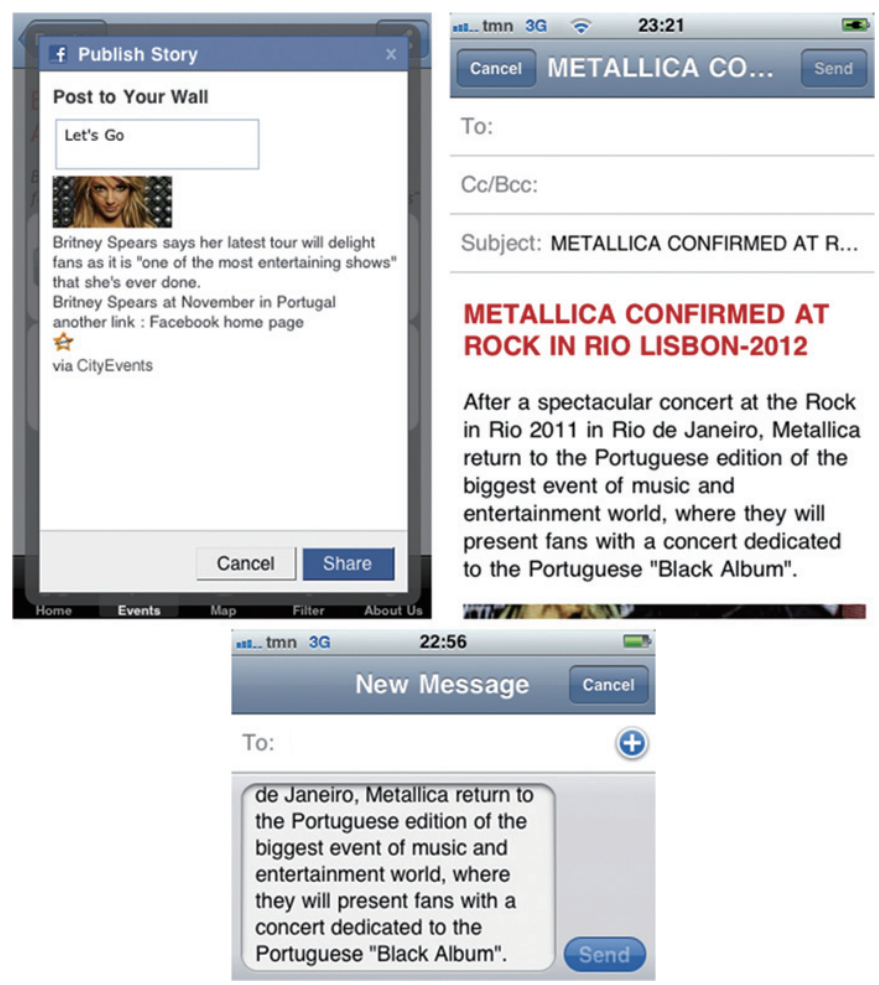

Fig. 8. Sharing events.

\section{Performance evaluation and results}

The proposed application should be experimented by real users in order to evaluate their experience. Then, a survey was proposed to support this study. A total of 114 users, $65 \%$ male and $45 \%$ female users from the University of Beira Interior, Portugal have answered the survey. The users have used the system for some time to become familiar with it in order to make a good experiment. After some experiments, they filled the CityEvents survey. The survey questions are available in Table 2 and the results may be found in Figs 10 and 11. It can be seen that the majority of the users agree the CityEvents application has an attractive design, it is user friendly and intuitive, presents a good navigation, and the options are clear and easy to use. The event detail is consistent and the text is easy to understand, fonts and all the event detail are easy to read on the screen. A large percentage of users also consider the application is very easy to use and helpful to bring the best events to him/her. The most part of the users agrees the application storing the users preferences and sort the events based on their preferences is good. Some users also agree that map view (with all the points of interests and events based on their preferences) is useful and attractive. Mostly users agree that events scored based on their preferences and location are useful.

Another issue is the distribution of the location-based scoring. Considering the news are usually distributed relative to an area of interest (for example, a country or a smaller area), high oscillations in the number of documents per relevance can be found rise to further optimizations. Thus, the distribution of nine sets of documents categorized by Accommodation, Leisure, Attractions, Community, Car, Food, Shopping, Supermarket, and Travel were analyzed and it was calculated the number of documents per 


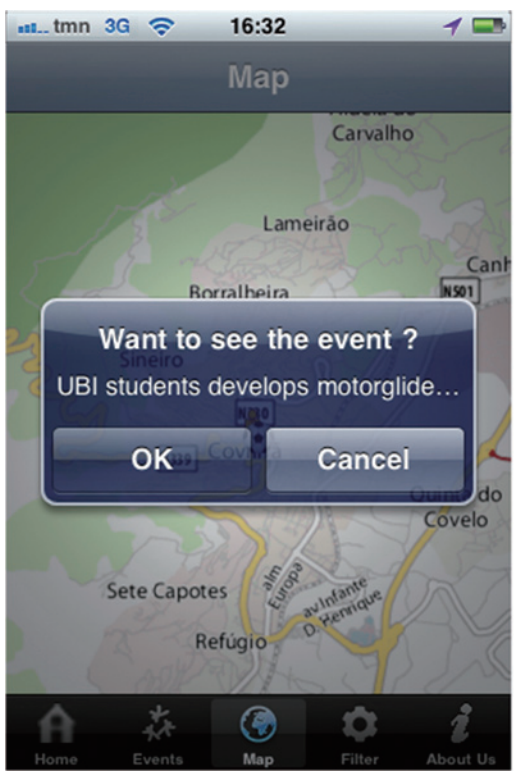

(a)

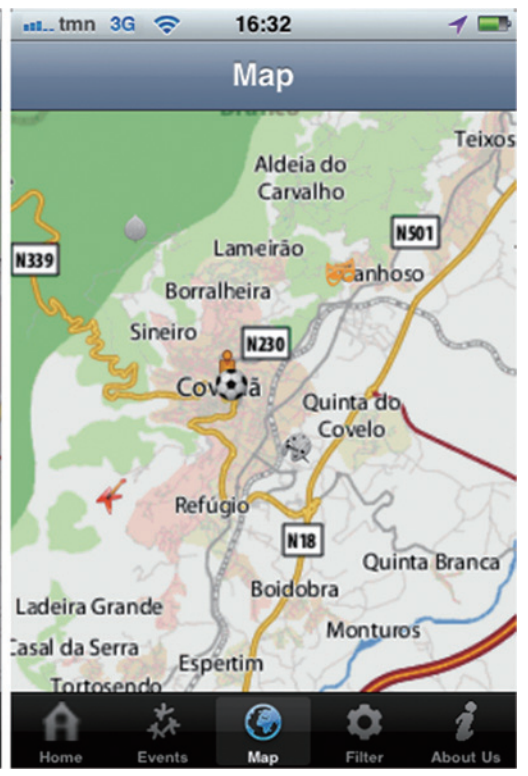

(b)

Fig. 9. Maps with alert of event and scoring events.

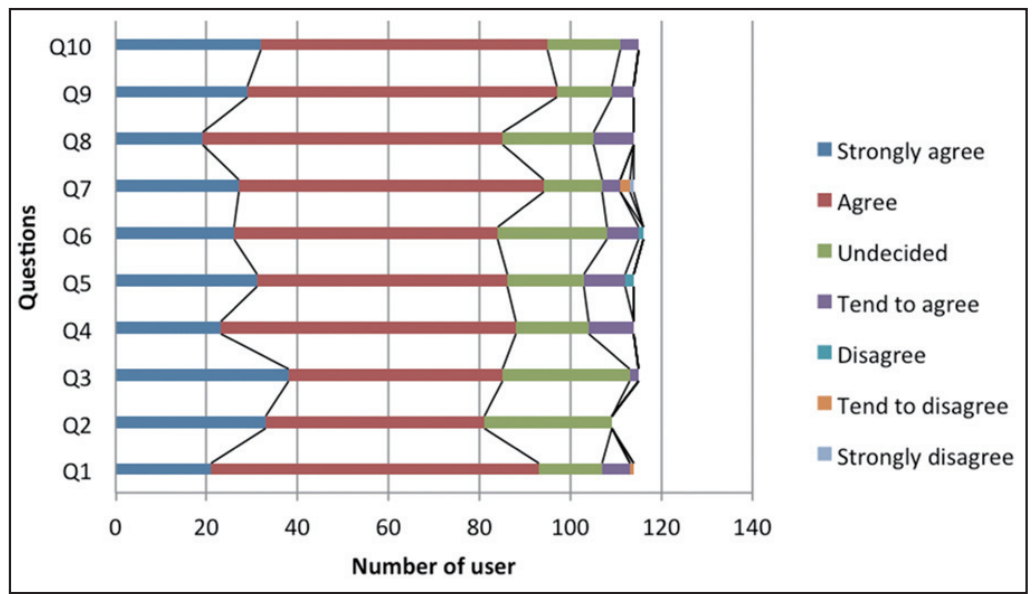

Fig. 10. Results of CityEvents users survey.

relevance windows. Figure 12 shows the results of the three categories with more documents and the average over all the nine categories, with a windowing width of 0.05 , leading to a resolution of 20 intervals in the range $[0,1]$.

The results of Fig. 12 clearly shown they are clustered around the chosen point of reference (the center of the bigger city in the area of interest) and the distribution curve is rather smooth, keeping almost a slowly increasing value between 0.10 and 0.85 . At higher resolutions (window width 0.1 , with 100 intervals in the range) there are also not high oscillations, as hypothesized. Thus, the study on this direction was stopped. 


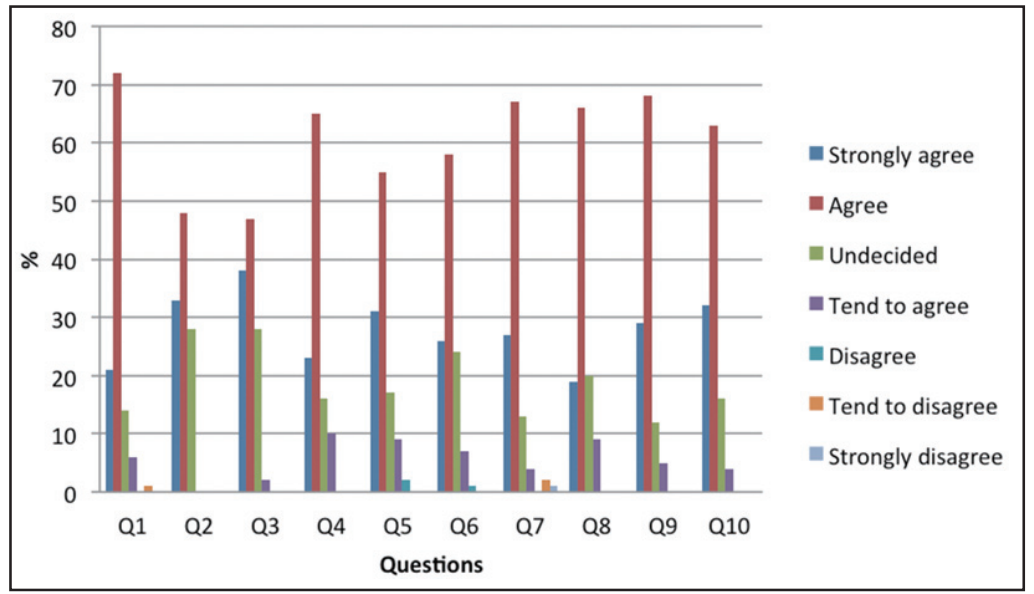

Fig. 11. Results of the users survey (percentages).

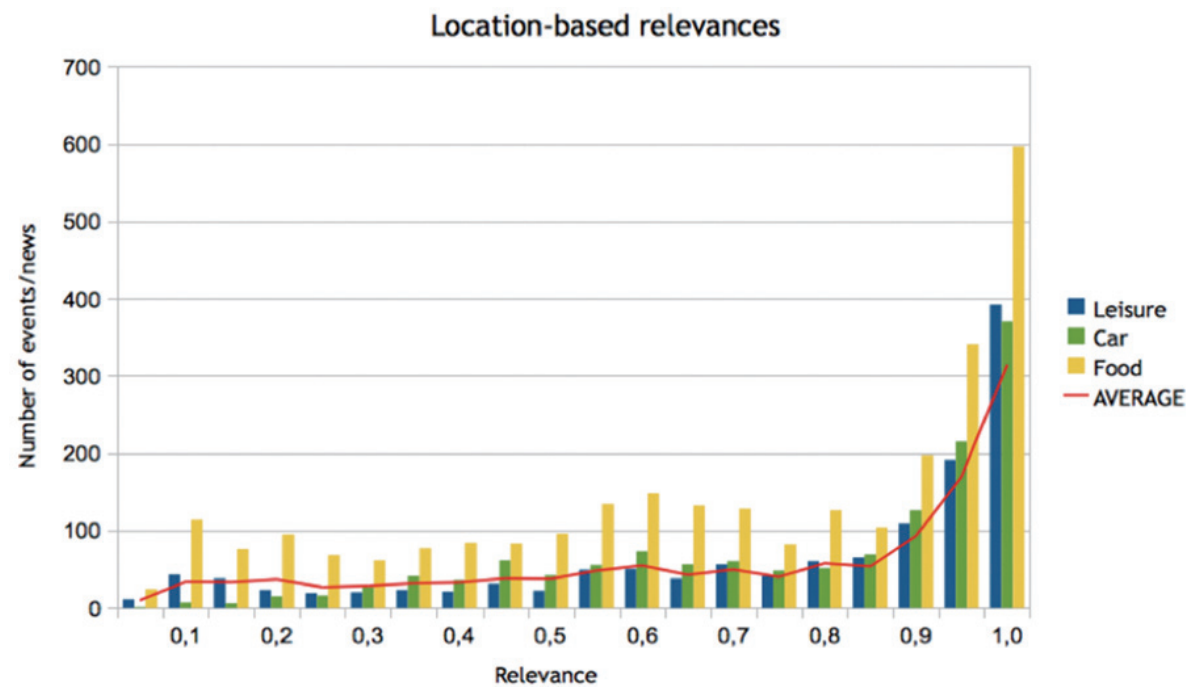

Fig. 12. Location-based relevance average.

The proposed re-normalization techniques and data-fusion equations are not intensive in terms of resources, processor or memory, and are by far less problematic than the re-sorting algorithms to be utilized in the content-adaptation layer of the application, or the display of the chosen news/events as points in a map on the mobile device.

\section{Conclusion and future work}

This paper proposed a ubiquitous location, habit, and context-aware mobile recommendation system including a client-side application called CityEvents and a Internet-based service providing sorted multimedia Geolocated cultural events to display on mobile devices. The user receives the best information, filtered and sorted the results based on user habits, context, location, and potentially other pa- 
rameters useful to achieve sharper results, in a personalized and interactive way. Different equations for re-normalization and data-fusion were evaluated and deployed in a complete demonstrative mobile application to study users experience. Some research directions were stopped when results from experimental data were available, while the majority of improvements and ideas developed during this work are suitable for further research.

As a future work, some improvements and research directions are suggested as follows: evaluating the effect of using more advanced data-fusion techniques; evaluating the effect of more complex taxonomies endowed with proper metrics; evaluating the effect of rank-based scoring instead of relevance-based scoring; considering documents as structured entities to identify some interesting relevance parameters; studying possibilities to use generic browsing history to generate some interesting relevance parameters; and the migration of the proposed mobile solution to other mobile platforms like Android or Windows Mobile.

\section{Acknowledgments}

This work has been partially supported by the Instituto de Telecomunicações, Next Generation Networks and Applications Group (NetGNA), Portugal, by National Funding from the FCT - Fundação para a Ciência e a Tecnologia through the PEst-OE/EEI/LA0008/2011 Project, and by the company Zirak s.r.l., Italy.

\section{References}

[1] A.K. Dey and G.D. Abowd, Towards a Better Understanding of Context and Context-Awareness, College of Computing, Georgia Institute of Technology, Atlanta GA USA, Technical Report GITGVU-99-22, 1999.

[2] A.K. Dey, Understanding and using context, Personal Ubiquitous Computing 5(1) 2001, 4-7.

[3] Apps Portugal - Appetite [Online]. Available: http://appsportugal.com/app/appetite-iphone/100 [Accessed: October 2012].

[4] B.M.C. Silva, P.A.C.S. Neves, M.K. Denko and J.J.P.C. Rodrigues, MP-Collaborator: A mobile collaboration tool in pervasive environment, in: 2009 IEEE International Conference on Wireless and Mobile Computing, Networking and Communications (WiMob 2009), Marrakech, Morocco, October 12-14, 2009, pp. 344-349.

[5] C.A. da Costa, A.C. Yamin and C.F.R. Geyer, Toward a general software infrastructure for ubiquitous computing, IEEE Pervasive Computing 7(1) (2008), 64-73.

[6] C. Wolfram, Q\&A: Conrad Wolfram on communicating with apps in Web 3.0 [Online]. Available: http://www.itpro. co.uk/621535/q-a-conrad-wolfram-on-communicating-with-apps-in-web-3-0 [Accessed: October 2012].

[7] C. Dobre, Context-aware platform for integrated mobile services, in: 2011 International Conference on Emerging Intelligent Data and Web Technologies (EIDWT), September 7-9, 2011, pp. 198-203.

[8] D. Taniar and J. Goh, On mining movement pattern from mobile users, International Journal of Distributed Sensor Networks 3(1) (2007), 69-86.

[9] D. Quercia, N. Lathia, F. Calabrese, G. Di Lorenzo and J. Crowcroft, Recommending social events from mobile phone location data, in: 10th International Conference on Data Mining (ICDM) 2010 IEEE, December 13-17, 2010, pp. 971976.

[10] D. Wang and A.A. Abouzeid, On the cost of knowledge of mobility in dynamic networks: An information-theoretic approach, IEEE Transactions on Mobile Computing 11(6) (June 2012), 995-1006.

[11] E. Fox and J. Shaw, Combination of Multiple Searches, NIST SPECIAL PUBLICATION SP, National Institute of Standards \& Techonology, 1994, pp. 243-243.

[12] E. Pitoura and G. Samaras, Locating objects in mobile computing, IEEE Transactions on Knowledge and Data Engineering 13(4) (July/August 2001), 571-592.

[13] F. Chen, M.P. Johnson, Y. Alayev, A. Bar-Noy and T.F. La Porta, Who, when, where: Timeslot assignment to mobile clients, IEEE Transactions on Mobile Computing 11(1) (January 2012), 73-85.

[14] F.S. Tsai, M. Etoh, X. Xie, W.C. Lee and Q. Yang, Introduction to mobile information retrieval, Intelligent Systems, IEEE 25(1) (January-February 2010), 11-15. 
[15] G. Ortiz and A.G. de Prado, Mobile-aware Web services, in: 3rd International Conference on Mobile Ubiquitous Computing, Systems, Services, and Technologies (UBICOMM 2009), Sliema, Malta, October 11-16, 2009, pp. 65-70.

[16] G. Salton, E.A. Fox and H. Wu, Extended boolean information retrieval, Communications of the ACM 26 (1983), 10221036.

[17] I. Sharp, K. Yu and T. Sathyan, Positional accuracy measurement and error modeling for mobile tracking, Transactions on Mobile Computing, IEEE 11(6) (June 2012), 1021-1032.

[18] iPhone's Location - Aware Apps - Loopt's FriendFinder [Online]. Available: https://www.loopt.com/ [Accessed: October 2012].

[19] J.J.P.C. Rodrigues, M. Oliveira and B. Vaidya, New trends on ubiquitous mobile multimedia applications, EURASIP Journal on Wireless Communications and Networking (September 2010).

[20] J.J.P.C. Rodrigues, I.M.C. Lopes, B.M.C. Silva and I. de la Torre, A new mobile ubiquitous computing application to control obesity: SapoFit, in: Informatics for Health and Social Care, Informa Healthcare, New York, USA, 2012, pp. 117.

[21] K.S. Su, D. Chan and F.C. Chan, Navigation on mobile news, in: 2nd International Conference on Mobile Technology, Applications and Systems, November 15-17, 2005, pp. 6-6.

[22] K.B. Ng and P.B. Kantor, An investigation of the preconditions for effective data fusion in ir: A pilot study, in: Proceedings of the 61th Annual Meeting of the American Society for Information Science, 1998.

[23] L. Guan, X. Ke, M. Song and J. Song, A survey of research on mobile cloud computing, in: 2011 IEEE/ACIS 10th International Conference on Computer and Information Science (ICIS 2011), May 16-18, 2011, pp. 387-392.

[24] L. Jessupand and D. Robey, The relevance of social issues in ubiquitous computing environments, Communications of the ACM 45(12) (December 2002), 88-91.

[25] L. Joon Ho, Analyses of multiple evidence combination, in: Proceedings of the 20th annual international ACM SIGIR conference on Research and development in information retrieval (SIGIR '97), ACM, New York, 1997, pp. 267-276.

[26] M. Weiser, The World is not a Desktop, ACM Interactions Magazine 1(1) (January 1994), pp. 7-8.

[27] M. Weiser, The computer for the 21st century, ACM SIGMOBILE Mobile Computing and Communications Review 3(3) (1999).

[28] M. Satyanarayanan, Pervasive computing: Vision and challenges, IEEE Personal Communications 8(4) (August 2001), $10-17$.

[29] M. Weiser and J. Seely Brown, The coming age of calm technology, Xerox PARC, October 5, 1996 [Online]. Available: http://www.ubiq.com/hypertext/weiser/acmfuture2endnote.htm [Accessed: October 2012].

[30] M. Weiser, Some computer science issues in ubiquitous computing, Commun ACM 36(7) (1993), 75-84.

[31] N. Verma, D. Mahajan, S. Sellamanickam and V. Nair, Learning hierarchical similarity metrics, in: IEEE Conference on Computer Vision and Pattern Recognition (CVPR) 2012, June 16-21, 2012, pp. 2280-2287.

[32] P.M.P. Rosa, J.A. Dias, I.C. Lopes, Rodrigues, J.J.P.C. Rodrigues and K. Lin, An ubiquitous mobile multimedia system for events agenda, in: Wireless Communications and Networking Conference (WCNC), 2012 IEEE, April 1-4, 2012, pp. 2103-2107.

[33] Q.H. Mahmoud and P. Popowicz, A mobile application development approach to teaching introductory programming, in: Frontiers in Education Conference (FIE), 2010 IEEE, October 27-30, 2010, pp. T4F-1-T4F-6.

[34] R. Ling, The Mobile Connection: The Rise of a Wireless Communications Society, Morgan Kaufmann Publishers, USA, 2004, pp. 83-121.

[35] S.J. Hong, J.Y.L. Thong, J.Y. Moon and K.Y. Tam, Understanding the behavior of mobile data services consumers, Information Systems Frontiers 10 (2008), 431-445.

[36] S.K. Mostefaoui, Z. Maamar and G.M. Giaglis, Advances in Ubiquitous Computing: Future Paradigms and Directions, IGI Publishing, New York, 2008.

[37] S. Pohl, A. Moffat and J. Zobel, Efficient extended boolean retrieval, IEEE Transactions on Knowledge and Data Engineering 24(6) (June 2012), 1014-1024.

[38] S. Quebe, J. Campbell, S. DeVilbiss and C. Taylor, Cooperative GPS navigation, Position Location and Navigation Symposium (PLANS), 2010 IEEE/ION, Indian Wells, CA, USA, May 4-6, 2010, pp. 834-837.

[39] S. Ja-Hwung, Y. Hsin-Ho, P.S. Yu and V.S. Tseng, Music Recommendation Using Content and Context Information Mining, IEEE Intelligent Systems 25(1) ( January 2010), 16-26.

[40] T. Teng Chen and D.C. Yen, Technical research themes of the mobile ubiquitous computing, in: Eighth International Conference on Mobile Business (ICMB 2009), June 27-28, 2009, pp. 221-226.

[41] The Mac Box - NearPics [Online]. Available: http://themacbox.co.uk/nearpics/ [Accessed: October 2012].

[42] Tweakersoft - AroundMe [Online]. Available: http://www.aroundmeapp.com/ [Accessed: October 2012].

[43] V. Santos, Applying social paradigms in mobile context-aware computing, 6th Iberian Conference on Information Systems and Technologies (CISTI), 2011, June 15-18, 2011, pp. 1-7.

[44] W.M.Y.W. Bejuri, M.M. Mohamad and M. Sapri, Ubiquitous positioning: A taxonomy for location determination on mobile navigation system, International Journal Signal \& Image Processing (SIPIJ) 2(1) (March 2011). 
[45] Weather for Apple iPhone Smartphones - WeatherBug [Online]. Available: http://weather.weatherbug.com/mobile/ weatherbug-for-iphone.html [Accessed: October 2012].

[46] Y. Bejerano, Coverage verification without location information, IEEE Transactions on Mobile Computing 11(4) (April 2012), 631-643.

[47] Y. Özgün and C. E. Rıza, iConAwa-An intelligent context-aware system, Expert Systems with Applications 39(3) (2012), 2907-2918, Elsevier.

Pedro M. Pinto Rosa received his Master degree in Informatics Engineering from University of Beira Interior, 2012 under the supervision of Professor Joel Rodrigues. He is an MSc member of the Next Generation Networks and Applications Group (NetGNA) at the Instituto de Telecomunicações, Portugal since March 2009. His main research topics include wireless sensor networks and modeling tools, mobile and ubiquitous computing. He has authored or co-authored of several papers in international journals and conferences.

Joel J. P. C. Rodrigues is a professor at the University of Beira Interior (UBI), Covilhã, Portugal, and researcher at the Instituto de Telecomunicações, Portugal. He received a $\mathrm{PhD}$ degree in informatics engineering, an MSc degree from the University of Beira Interior, and a five-year BSc degree (licentiate) in informatics engineering from the University of Coimbra, Portugal. $\mathrm{He}$ is the Director of the Master degree in Informatics Engineering at UBI. He is the leader of NetGNA Research Group (http://netgna.it.ubi.pt), the Chair of the IEEE ComSoc Technical Committee on Communications Software, the Vice-Chair of the IEEE ComSoc Technical Committee on eHealth, and Member Representative of the IEEE Communications Society on the IEEE Biometrics Council. He is the editor-in-chief of the International Journal on E-Health and Medical Communications, the editor-in-chief of the Recent Patents on Telecommunications, and editorial board member of several international journals. $\mathrm{He}$ has been general chair and TPC Chair of many international conferences. He is a member of many international TPCs and participated in several international conferences organization. He has authored or coauthored over 240 papers in refereed international journals and conferences, a book, and 2 patents. He had been awarded the Outstanding Leadership Award of IEEE GLOBECOM 2010 as CSSMA Symposium Co-Chair and several best papers awards. Prof. Rodrigues is a licensed professional engineer (as senior member), member of the Internet Society, an IARIA fellow, and a senior member of ACM and IEEE.

Filippo Basso received his 4-year M.Sc. degree cum laude in General Mathematics in 1999, from the University of Pisa and the Science Faculty degree cum laude in the same year from Scuola Normale Superiore di Pisa. He worked in Research and Development since 2000 in Zirak s.r.l., Italian SME, where focused on Information Retrieval, eHealth, Mobile and Ubiquitous Computing and generic modelling, algorithms and optimizations. His research interests comprehend theoretical frontiers topics and their multi-disciplinary applications. 

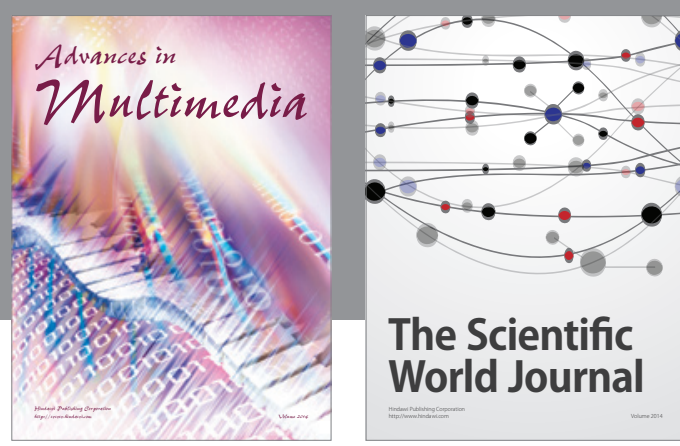

The Scientific World Journal
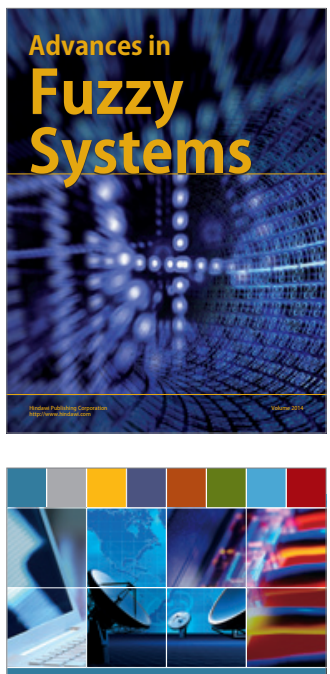

Computer Networks and Communications
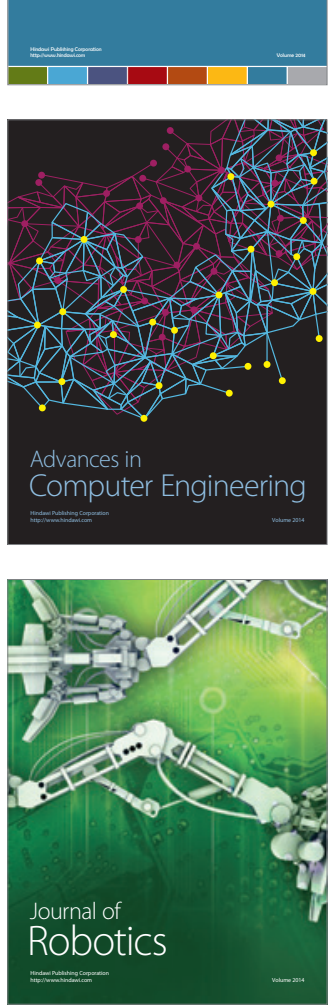
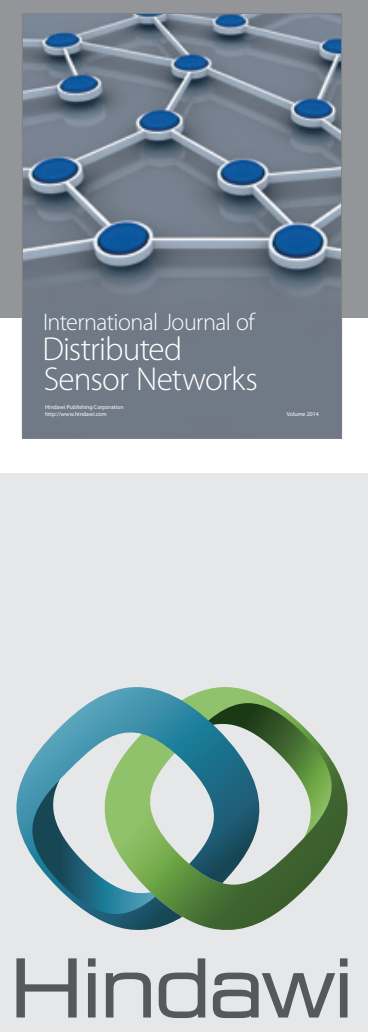

Submit your manuscripts at

http://www.hindawi.com
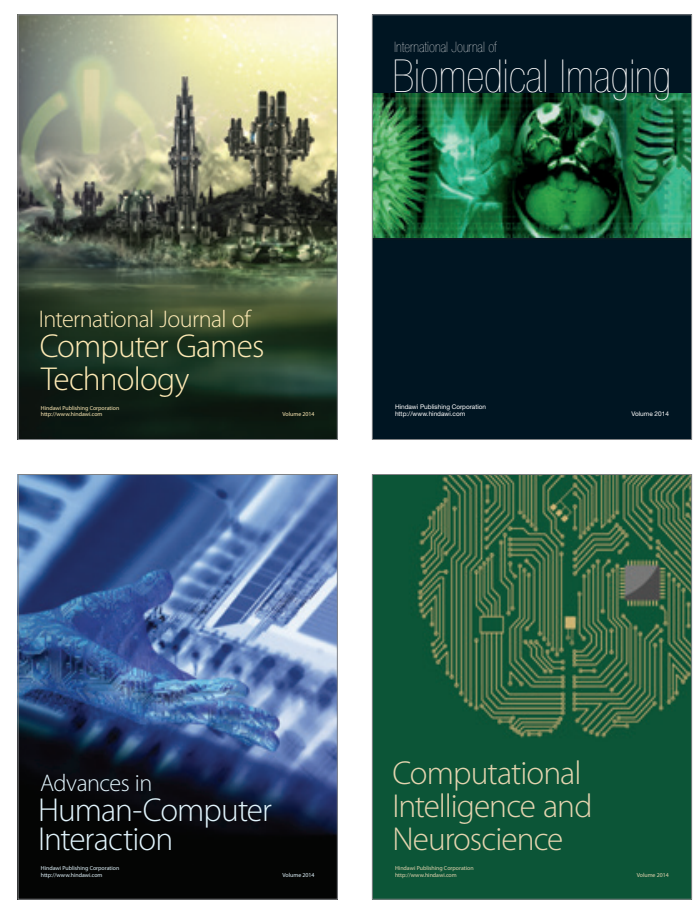
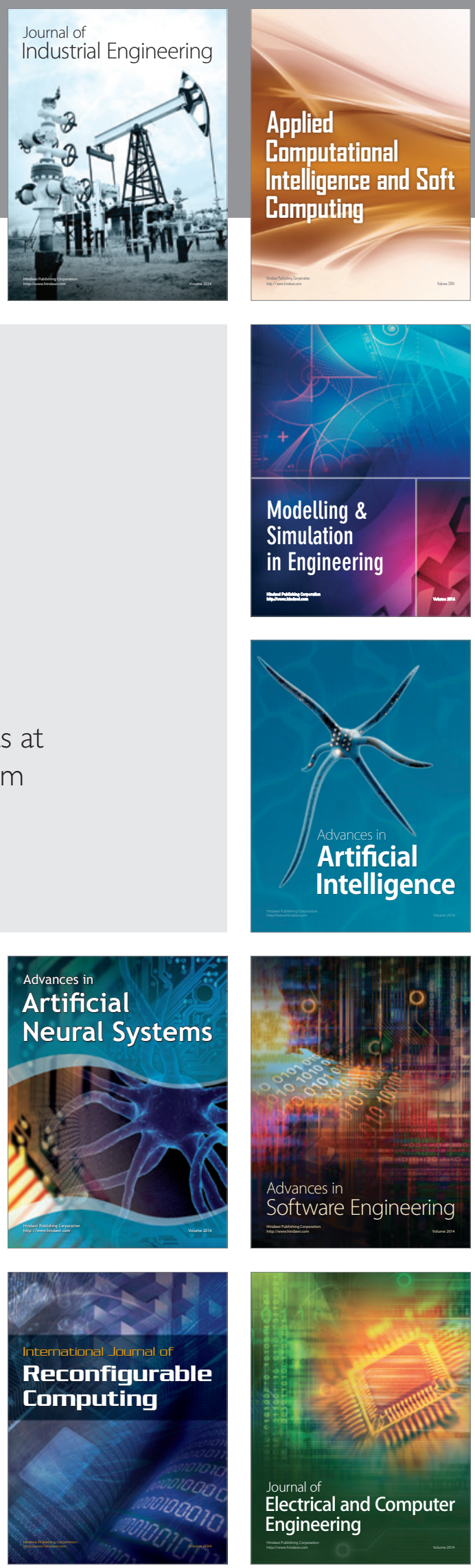